Published in final edited form as:

Thromb Res. 2016 January ; 137: 97-102. doi:10.1016/j.thromres.2015.11.009.

\title{
Assessment of Algorithms to Identify Patients with Thrombophilia following Venous Thromboembolism
}

\author{
Thomas Delate, PhD, MS ${ }^{a, b, \wedge, *}$, Wendy Hsiao, BS ${ }^{c,}$, Benjamin Kim, MD, MPhil ${ }^{d}$, Daniel M. \\ Witt, PharmD ${ }^{a}, \mathrm{e}$, Melissa R. Meyer, PharmD ${ }^{\mathrm{a}}$, Alan S. Go, MD $^{\mathrm{f} g, \mathrm{~h}}$, and Margaret C. Fang, MD, \\ $\mathrm{MPH}^{\mathrm{i}}$ \\ aDepartment of Clinical Pharmacy, Kaiser Permanente Colorado. Affiliation Address: 16601 East \\ Centretech Parkway, Aurora, CO 80011, USA \\ bDepartment of Pharmacy, Skaggs School of Pharmacy and Pharmaceutical Sciences, University \\ of Colorado, Denver. Affiliation Address: 12850 East Montview Boulevard, Aurora, CO 80045 \\ 'Keck School of Medicine, University of Southern California. Affiliation Address: 1975 Zonal Ave, \\ Los Angeles, CA 90033, USA \\ ${ }^{d}$ Division of Hematology/Oncology, Department of Medicine, University of California, San \\ Francisco. Affiliation Address: 505 Parnassus Ave., M1286, Box 1270, San Francisco, CA 94143, \\ USA
}

eDepartment of Pharmacotherapy, University of Utah College of Pharmacy. Affiliation Address: 30 South 2000 East, Room 4926, Salt Lake City, UT 84112

'Division of Research, Kaiser Permanente of Northern California, Oakland, California. Affiliation Address: 2000 Broadway, Oakland, CA 94612, USA

gDepartments of Epidemiology, Biostatistics, and Medicine, University of California, San Francisco, San Francisco, California. Affiliation Address: 2000 Broadway, Oakland, CA 94612, USA

hDepartment of Health Research and Policy, Stanford University School of Medicine, Palo Alto, California. Affiliation Address: 2000 Broadway, Oakland, CA 94612, USA

iDivision of Hospital Medicine, Department of Medicine, University of California, San Francisco. Affiliation Address: 533 Parnassus Ave., Box 0131, Room U135, San Francisco, CA 94143, USA

\section{Abstract}

Introduction-Routine testing for thrombophilia following venous thromboembolism (VTE) is controversial. The use of large datasets to study the clinical impact of thrombophilia testing on patterns of care and patient outcomes may enable more efficient analysis of this practice in a wide range of settings. We set out to examine how accurately algorithms using International

^Corresponding author: Thomas Delate, PhD, MS; Address: 16601 East Centretech Parkway, Aurora, CO 80011; tom.delate@kp.org; Phone: 303-739-3538; Fax: 303-739-3574.

*Contributed equally to this work

Presented as a poster at the $56^{\text {th }}$ American Society of Hematology Annual Meeting and Exposition, San Francisco, CA, December 69, 2014. 
Classification of Diseases $9^{\text {th }}$ Revision (ICD-9) codes and/or pharmacy data reflect laboratoryconfirmed thrombophilia diagnoses.

Materials and Methods-A random sample of adult Kaiser Permanente Colorado patients diagnosed with unprovoked VTE between 1/2004 and 12/2010 underwent medical record abstraction of thrombophilia test results. Algorithms using "ICD-9" (positive if a thrombophilia ICD-9 code was present), "Extended anticoagulation (AC)" (positive if AC therapy duration was $>6$ months), and "ICD-9 \& Extended AC" (positive for both) criteria to identify possible thrombophilia cases were tested. Using positive thrombophilia laboratory results as the gold standard, the sensitivity, specificity, positive predictive value (PPV), and negative predictive value of each algorithm were calculated, along with $95 \%$ confidence intervals (CIs).

Results-In our cohort of 636 patients, sensitivities were low $(<50 \%)$ for each algorithm. "ICD-9" yielded the highest PPV (41.5\%, 95\% CI 26.3-57.9\%) and a high specificity (95.9\%, 95\% CI 94.0-97.4\%). "Extended AC" had the highest sensitivity but lowest specificity, and "ICD-9 \& Extended AC" had the highest specificity, but the lowest sensitivity.

Conclusions-ICD-9 codes for thrombophilia are highly specific for laboratory-confirmed cases, but all algorithms had low sensitivities. Further development of methods to identify thrombophilia patients in large datasets is warranted.

\section{Keywords}

Thrombophilia; venous thromboembolism; dataset; algorithms; sensitivity; specificity

\section{Introduction}

Routine testing for thrombophilia following venous thromboembolism (VTE) is controversial [1,2]. Randomized clinical trials or observational studies to-date have not demonstrated a reduced risk of recurrent VTE associated with thrombophilia testing [3, 4]. Current guidelines recommend thrombophilia testing only if the results are likely to influence treatment decisions and usually only in the setting of unprovoked VTE $[5,6]$.

Analysis of thrombophilia testing in large VTE datasets may enable evaluation of quality of care and clarification of issues surrounding thrombophilia testing (e.g., clinical utility, impact on outcomes). The only large, prospective, observational VTE cohort studies evaluating thrombophilia testing come from the RIETE initiative [7-13], which utilized detailed inpatient and outpatient medical record abstraction not easily replicated in other settings. Utilization of administrative datasets to assess the impact of thrombophilia offers the ability to study real-world patterns of care and patient outcomes in large numbers. Positive predictive values (PPVs) of approximately 95\% have been achieved using International Classification of Diseases $9^{\text {th }}$ Revision (ICD-9) codes to identify patients with VTE in a large dataset [14]. However, ICD-9 codes have not been evaluated similarly to identify patients with thrombophilia. The goal of this study was to evaluate whether patients with unprovoked VTE and laboratory-confirmed thrombophilia can be efficiently identified in a large dataset with high sensitivity and specificity (>90\%) using algorithms based on ICD-9 codes and/or electronic pharmacy records. 


\section{Materials and Methods}

\section{Patients and Study Period}

Kaiser Permanente Colorado (KPCO) patients were identified as part of the Cardiovascular Research Network Venous Thromboembolism (CVRN VTE) Study. The CVRN VTE Study identified all patients $\geq 21$ years of age with an ICD-9 primary or secondary diagnosis code of VTE in the time period between January 1, 2004 and December 31, 2010 who had at least 180 consecutive days of health plan membership prior to the event (index VTE). Index VTE events were categorized as pulmonary embolism (PE), upper or lower extremity deep vein thrombosis (DVT), or other venous thrombosis (codes available upon request). Both inpatient and outpatient events were included. For this study, patients with atrial fibrillation, prior VTE or warfarin prescription <3 years prior to index VTE event, and recurrent VTE during the study period were excluded to ensure selection of patients who received anticoagulation (AC) for their initial VTE event. Patients with $<1$ month of continuous health plan enrollment and prescription drug benefit after index VTE were also excluded, as complete data regarding patients' AC treatment for their VTE event was desired. Finally, patients with provoked VTE (active cancer, surgery $<1$ month prior to index VTE event, or pregnancy $<1$ year prior to index VTE event) were excluded as these patients were less likely to undergo thrombophilia testing. We included patients who had non-surgical trauma in the month prior to their VTE diagnosis based on the previous finding that this risk factor independently predicted having had a positive thrombophilia test result (Meyer-submitted). Patients were followed for up to 1 year after their index VTE. This study was reviewed and all aspects approved by the KPCO Institutional Review Board.

\section{Data Collection}

A random sample of KPCO CVRN VTE patients underwent medical record review using a structured data collection form to determine whether the events were valid, acute VTE events. Thrombophilia laboratory test results were extracted from KPCO's electronic laboratory database and confirmed with manual chart review as necessary. Tests included factor V Leiden, prothrombin gene mutation, antithrombin activity, protein $\mathrm{C}$ activity, protein S activity, and antiphospholipid antibody syndrome (APS) tests (lupus anticoagulant [hexagonal phase and Russell's viper venom time], Cardiolipin immunoglobulin $[\mathrm{Ig}] \mathrm{G}$, and $\beta$-2 glycoprotein $\operatorname{IgG}$ ). Testing for APS was considered positive if there were two positive APS results separated by at least 6 weeks. Patients were considered "positive" for laboratory-confirmed thrombophilia if $\geq 1$ test was positive, "negative" if no tests were positive, and "not tested" if none of the abstracted thrombophilia laboratory tests were performed. Because guidelines recommend that providers who do not suspect thrombophilia should not order thrombophilia testing for their patients [15], we chose to combine patients who tested negative for thrombophilia with those who did not undergo testing ("no thrombophilia") for our analyses. Duration of AC was determined from electronic pharmacy records.

\section{Thrombophilia Identification Algorithms}

The study cohort was subjected to "ICD-9" (positive: $\geq 1$ ICD-9 code for primary [289.81] or secondary hypercoagulable state [289.82], negative otherwise) and "extended AC" criteria 
(positive: received AC $>6$ months duration after index VTE, negative otherwise) individually and in combination ("ICD-9 \& extended AC") to identify possible thrombophilia cases (Figure 1).

\section{Statistical Analyses}

Using positive thrombophilia laboratory test results as the gold standard, sensitivities, specificities, PPVs, and negative predictive values (NPVs) of ICD-9, extended AC, and ICD-9 \& extended AC identification strategies were calculated along with binomial proportion $95 \%$ confidence intervals (CIs). Patient characteristics were analyzed overall and by thrombophilia test result and testing statuses. Differences in proportions and means were assessed between groups using the chi-square or Fisher's exact test, as appropriate, or independent sample t-test, respectively. Analyses were performed with SAS 9.2 (SAS Institute Inc., Cary, NC).

\section{Results}

\section{Patient Characteristics}

There were 1314 patients with confirmed index VTE. We excluded 678 patients who had recurrent VTE, atrial fibrillation, cancer, surgery, pregnancy, or $<1$ month enrollment after VTE during the study period, leaving 636 patients to be included in the analysis (Figure 2). Of these patients, 206 (32.4\%) were tested for thrombophilia; 48 (7.5\%) had at least one positive thrombophilia test result, $158(24.8 \%)$ tested negative, and $430(67.6 \%)$ were not tested (588 [92.5\%] patients were considered to have "no thrombophilia"; Table 1).

The overall cohort had a mean (standard deviation) age of 62.7 (17.0) years, $49.5 \%$ were male, and $55.2 \%$ and $54.4 \%$ had a lower extremity DVT and PE, respectively. After applying the various algorithms, $6.5 \%$ percent had at least one ICD-9 thrombophilia code while $47.6 \%$ received extended AC following VTE. Seventeen of 48 (35.4\%) thrombophiliapositive patients versus 15 of 158 thrombophilia-negative (9.5\%) and 9 of $430(2.1 \%)$ not tested patients had at least one ICD-9 thrombophilia code $(p<0.001)$. The percentage of patients receiving extended $\mathrm{AC}$ was slightly less than $50 \%$, regardless of their thrombophilia test status or results (Table 1).

There were statistically significant differences in the mean age ( $p=0.001)$, proportion with family history of VTE $(p=0.001)$, proportion with at least one thrombophilia test ordered $(p<0.001)$, and proportion with presence of at least one ICD-9 thrombophilia code $(p<0.001)$ between the thrombophilia-positive and no thrombophilia groups. Patients who received thrombophilia testing were younger, had a family history of VTE, were taking hormone therapy at the time of index VTE event, and had at least one ICD-9 thrombophilia code (all $p<0.001)$ compared to non-tested patients (Table 1). Such differences were also seen between patients who tested negative versus those who were not tested (Table 1).

\section{Algorithm Performance Results}

Negative predictive values were high but sensitivities and PPVs were low in all three algorithms. Applying thrombophilia ICD-9 codes only yielded the numerically highest PPV 
(41.5\%, 95\% CI 26.3-57.9\%) and a high specificity (95.9\%, 95\% CI 94.0-97.4\%). The extended AC algorithm had the numerically highest sensitivity (45.8\%, 95\% CI 31.4-60.8\%) but the lowest specificity $(52.2 \%, 95 \%$ CI 48.1-56.3\%). The ICD-9 \& extended AC algorithm had the numerically highest specificity $(97.1 \%, 95 \%$ CI $95.4-98.3 \%)$ and a high NPV (93.6\%, 95\% CI 91.4-95.4\%), but the lowest sensitivity (18.8\%, 95\% CI 9.0-32.6\%)

(Table 2).

\section{Discussion}

In this study of 636 patients with unprovoked VTE, approximately one-third of the patients were tested for thrombophilia. The high specificity of our ICD-9 algorithm suggests that the presence of thrombophilia ICD-9 codes can be used to rule in patients with laboratoryconfirmed thrombophilia. Unfortunately, all algorithms had limited ability to capture the entire VTE population who underwent thrombophilia testing based on the observed low sensitivities and PPVs.

That there was no significant difference in proportions of patients who received extended AC after VTE between the thrombophilia-positive and no thrombophilia groups could suggest that thrombophilia test results were not associated with differences in treatment decisions regarding duration of $\mathrm{AC}$, though we were not able to obtain data on all confounding factors. Because so few patients in our cohort had laboratory-confirmed thrombophilia, we were unable to distinguish whether any one type of thrombophilia was associated with extended AC duration following an unprovoked VTE event. Providers likely choose extended AC for patients with unprovoked VTE because of the associated higher risk for VTE recurrence. Therefore, it appears that the decision to extend the duration of $\mathrm{AC}$ is not related to the presence or absence of a positive thrombophilia result and may instead be based on clinical grounds as guidelines recommend [16].

The low sensitivities of all three algorithms support that medical record/laboratory record abstraction captures more thrombophilia-positive patients than the tested algorithms. On the other hand, the high NPVs indicate that providers are not assigning thrombophilia ICD-9 codes or continuing AC beyond six months if the patient does not have laboratory-confirmed thrombophilia. It may be that providers are relying on the presence of a laboratoryconfirmed diagnosis of thrombophilia to label their patient as such, although just over a third of thrombophilia-positive patients had a corresponding ICD-9 code for thrombophilia. Of note, our algorithm results are comparable to other studies investigating the use of administrative data to identify patients with related conditions, such as heart failure [17] and obesity [18].

There are several limitations to this study. Laboratory evidence of APS was considered to be met if there were two positive APS test results separated by at least six weeks, according to the original Sapporo criteria published in 1999 [19], instead of by at least 12 weeks, as published in 2006 in the revised Sapporo criteria [20]. The original criteria were used because a significant proportion of the study population was diagnosed with unprovoked VTE from 2004 to 2006, prior to the dissemination of the revised Sapporo criteria. Also, given that we had so few patients who met the diagnostic laboratory criteria of APS, our 
overall results are unlikely to be affected significantly by the selection of this definition. IgM results for the Cardiolipin and $\beta-2$ glycoprotein tests were not included, as these were not required by the original Sapporo criteria. Some patients may have been incorrectly determined as thrombophilia-negative, although again, this likely impacts a very small number of patients in our study. Also, some patients did not undergo testing of all thrombophilia tests. Therefore, the proportion of unprovoked VTE patients who have a positive thrombophilia result is likely to be higher than what we are able to report, as thrombophilia testing by clinicians is oftentimes incomplete. We combined patients who were not tested with thrombophilia-negative patients. Although these two groups differed in baseline characteristics similar to how tested versus not tested patients differed, there is strong clinical rationale to group thrombophilia-negative and not tested patients together as neither can be considered to have thrombophilia based on the gold standard of positive test results. In addition, guidelines recommend that providers who do not suspect thrombophilia should not order thrombophilia testing for such patients [15], which further support this grouping.

\section{Conclusions}

This is the first study, to our knowledge, to evaluate if algorithms using ICD-9 codes and/or pharmacy claims data can accurately identify laboratory-confirmed cases of thrombophilia in a large cohort of unprovoked VTE patients. We found that thrombophilia test results were not associated with differences in the proportion of patients who received extended AC.

There appeared to be a lack of concordance between thrombophilia laboratory test result and ICD-9 codes, but ICD-9 codes for primary and secondary hypercoagulable state accurately identified individuals who had positive thrombophilia test results. In our thrombophilia identification algorithms, the low sensitivities and PPVs of algorithms using ICD-9 codes and/or pharmacy claims data suggest that medical record abstraction is still required to capture all cases of laboratory-confirmed thrombophilia. If the use of administrative data to investigate the implications of thrombophilia testing in large VTE cohorts is to be optimized, further development of methods to identify thrombophilia status in large datasets is warranted.

\section{Acknowledgments}

The research for this manuscript was funded by the National Heart, Lung, and Blood Institute grants U19 HL91179-01 (PI: Go) and R01 HL103820 (PI: Fang). Neither funding source was involved in study design; in the collection, analysis and interpretation of data; in the writing of the report; or in the decision to submit the article for publication.

\section{Abbreviations}
AC anticoagulation
APS antiphospholipid antibody syndrome
CIs confidence intervals

CVRN VTE Cardiovascular Research Network Venous Thromboembolism 


$\begin{array}{ll}\text { DVT } & \text { deep vein thrombosis } \\ \text { ICD-9 } & \text { International Classification of Diseases } 9^{\text {th }} \text { Revision } \\ \text { Ig } & \text { immunoglobulin } \\ \text { KPCO } & \text { Kaiser Permanente Colorado } \\ \text { NPV(s) } & \text { negative predictive value(s) } \\ \text { PE } & \text { pulmonary embolism } \\ \text { PPV(s) } & \text { positive predictive value(s) } \\ \text { RIETE } & \text { Registro Informatizado de Enfermedad TromboEmbolica } \\ \text { VTE } & \text { venous thromboembolism }\end{array}$

\section{References}

1. Favaloro EJ, McDonald D, Lippi G. Laboratory investigation of thrombophilia: the good, the bad, and the ugly. Seminars in thrombosis and hemostasis. 2009; 35:695-710. [PubMed: 20013536]

2. Middeldorp S. Is thrombophilia testing useful? Hematology / the Education Program of the American Society of Hematology American Society of Hematology Education Program. 2011; 2011:150-155.

3. Cohn DM, Vansenne F, de Borgie CA, Middeldorp S. Thrombophilia testing for prevention of recurrent venous thromboembolism. The Cochrane database of systematic reviews. 2012; 12:CD007069. [PubMed: 23235639]

4. Coppens M, Reijnders JH, Middeldorp S, Doggen CJ, Rosendaal FR. Testing for inherited thrombophilia does not reduce the recurrence of venous thrombosis. Journal of thrombosis and haemostasis : JTH. 2008; 6:1474-1477. [PubMed: 18540999]

5. Baglin T, Gray E, Greaves M, Hunt BJ, Keeling D, Machin S, et al. Clinical guidelines for testing for heritable thrombophilia. British journal of haematology. 2010; 149:209-220. [PubMed: 20128794]

6. Chong LY, Fenu E, Stansby G, Hodgkinson S. Guideline Development G. Management of venous thromboembolic diseases and the role of thrombophilia testing: summary of NICE guidance. Bmj. 2012; 344:e3979. [PubMed: 22740565]

7. Blanco-Molina A, Trujillo-Santos J, Criado J, Lopez L, Lecumberri R, Gutierrez R, et al. Venous thromboembolism during pregnancy or postpartum: findings from the RIETE Registry. Thrombosis and haemostasis. 2007; 97:186-190. [PubMed: 17264945]

8. Blanco-Molina A, Trujillo-Santos J, Tirado R, Canas I, Riera A, Valdes M, et al. Venous thromboembolism in women using hormonal contraceptives. Findings from the RIETE Registry. Thrombosis and haemostasis. 2009; 101:478-482. [PubMed: 19277408]

9. Gabriel F, Portoles O, Labios M, Rodriguez C, Cisneros E, Vela J, et al. Usefulness of thrombophilia testing in venous thromboembolic disease: findings from the RIETE registry. Clinical and applied thrombosis/hemostasis : official journal of the International Academy of Clinical and Applied Thrombosis/Hemostasis. 2013; 19:42-47. [PubMed: 22327823]

10. Gadelha T, Roldan V, Lecumberri R, Trujillo-Santos J, del Campo R, Poggio R, et al. Clinical characteristics of patients with factor V Leiden or prothrombin G20210A and a first episode of venous thromboembolism. Findings from the RIETE Registry. Thrombosis research. 2010; 126:283-286. [PubMed: 20627204]

11. Monreal M, Campo RD, Papadakis E. Thrombophilia and venous thromboembolism: RIETE experience. Best practice \& research Clinical haematology. 2012; 25:285-294. [PubMed: 22959545] 
12. Roldan V, Lecumberri R, Munoz-Torrero JF, Vicente V, Rocha E, Brenner B, et al. Thrombophilia testing in patients with venous thromboembolism. Findings from the RIETE registry. Thrombosis research. 2009; 124:174-177. [PubMed: 19101711]

13. Tsoran I, Saharov G, Brenner B, Barron M, Valdes V, de la Roca Toda M, et al. Prolonged travel and venous thromboembolism findings from the RIETE registry. Thrombosis research. 2010; 126:287-291. [PubMed: 20624636]

14. White RH, Garcia M, Sadeghi B, Tancredi DJ, Zrelak P, Cuny J, et al. Evaluation of the predictive value of ICD-9-CM coded administrative data for venous thromboembolism in the United States. Thrombosis research. 2010; 126:61-67. [PubMed: 20430419]

15. De Stefano V, Rossi E. Testing for inherited thrombophilia and consequences for antithrombotic prophylaxis in patients with venous thromboembolism and their relatives. A review of the Guidelines from Scientific Societies and Working Groups. Thrombosis and haemostasis. 2013; 110:697-705. [PubMed: 23846575]

16. Kearon C, Akl EA, Comerota AJ, Prandoni P, Bounameaux H, Goldhaber SZ, et al. Antithrombotic therapy for VTE disease: Antithrombotic Therapy and Prevention of Thrombosis, 9th ed: American College of Chest Physicians Evidence-Based Clinical Practice Guidelines. Chest. 2012; 141:e419S-e494S. [PubMed: 22315268]

17. McCormick N, Lacaille D, Bhole V, Avina-Zubieta JA. Validity of heart failure diagnoses in administrative databases: a systematic review and meta-analysis. PloS one. 2014; 9:e104519. [PubMed: 25126761]

18. Martin BJ, Chen G, Graham M, Quan H. Coding of obesity in administrative hospital discharge abstract data: accuracy and impact for future research studies. BMC health services research. 2014; 14:70. [PubMed: 24524687]

19. Wilson WA, Gharavi AE, Koike T, Lockshin MD, Branch DW, Piette JC, et al. International consensus statement on preliminary classification criteria for definite antiphospholipid syndrome: report of an international workshop. Arthritis Rheum. 1999; 42:1309-1311. [PubMed: 10403256]

20. Miyakis S, Lockshin MD, Atsumi T, Branch DW, Brey RL, Cervera R, et al. International consensus statement on an update of the classification criteria for definite antiphospholipid syndrome (APS). Journal of thrombosis and haemostasis : JTH. 2006; 4:295-306. [PubMed: 16420554] 

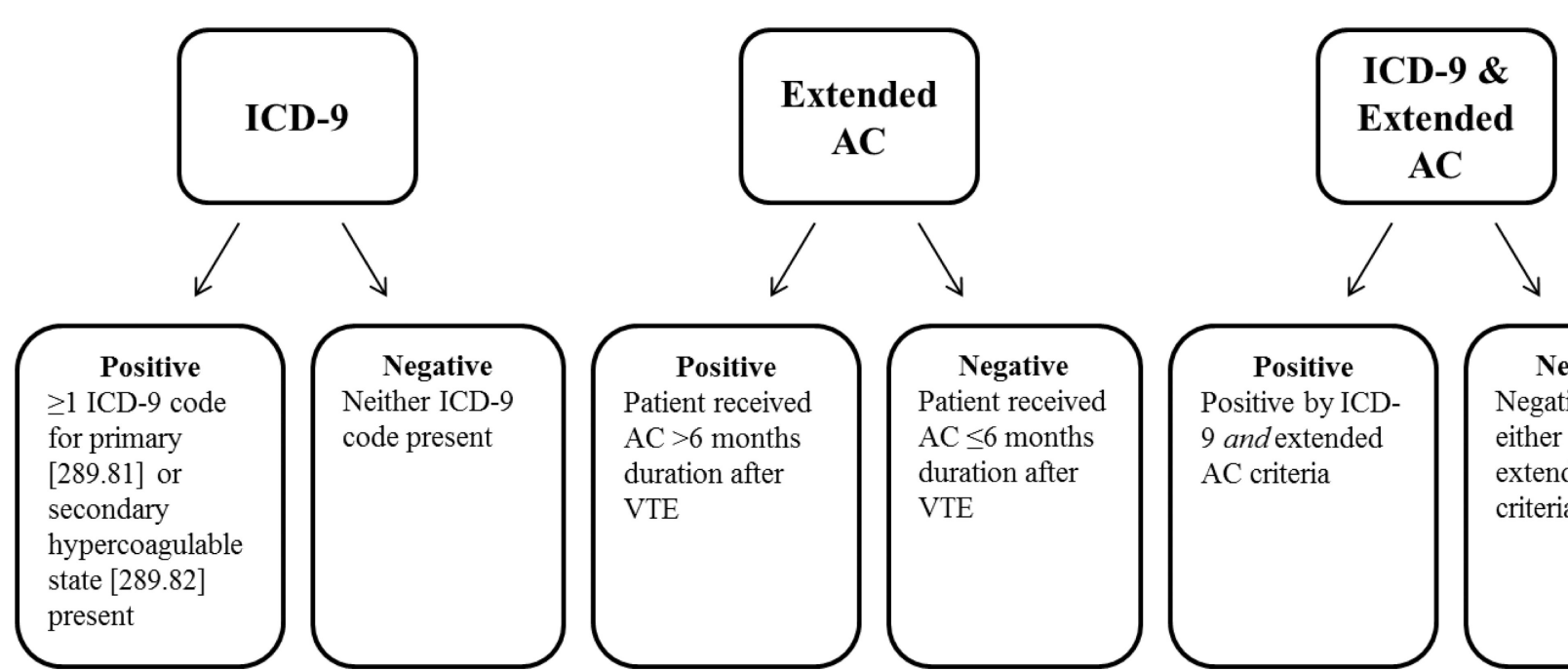

Figure 1. Criteria for Thrombophilia Status by Thrombophilia Identification Algorithms Abbreviations: AC, anticoagulation; ICD-9, International Classification of Diseases $9^{\text {th }}$ Revision; VTE, venous thromboembolism. 
Excluded

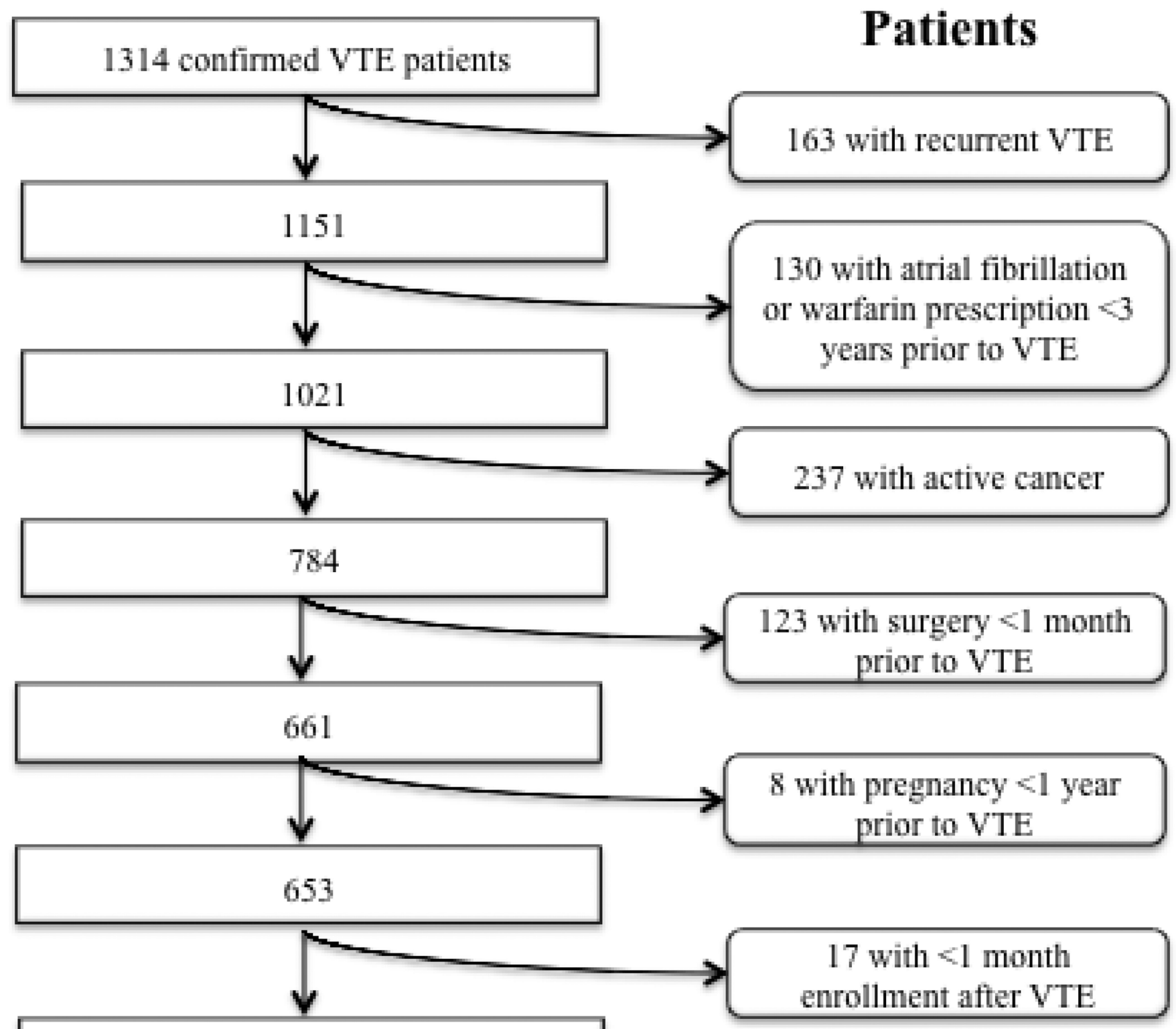

\section{6 study patients}

Figure 2. Analytic Cohort Selection

Abbreviations: VTE, venous thromboembolism. 


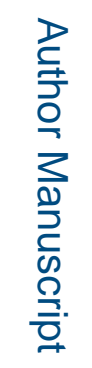

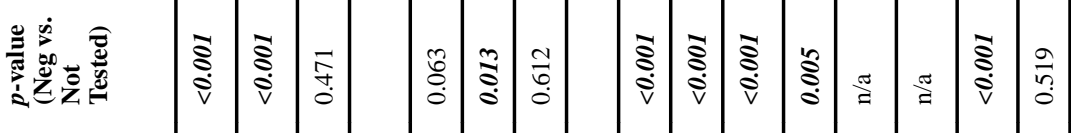

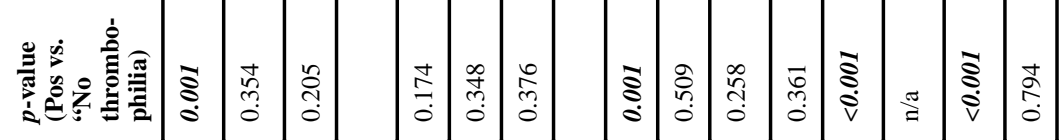

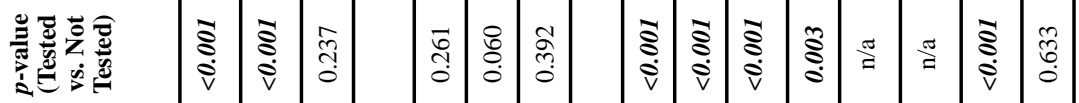

I

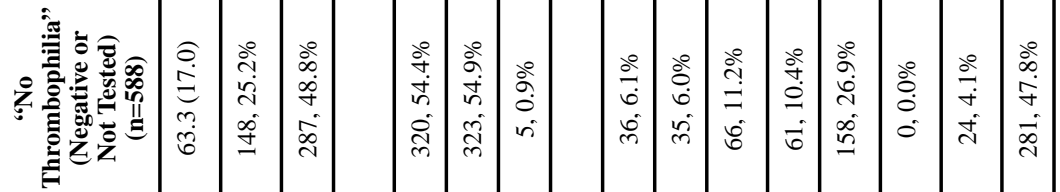

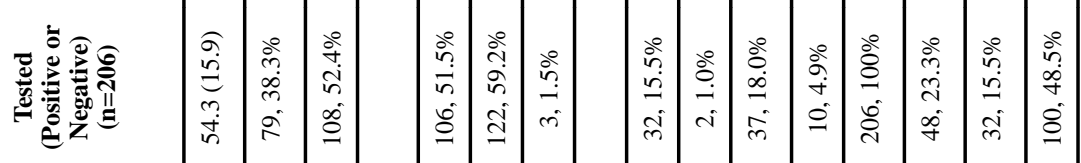

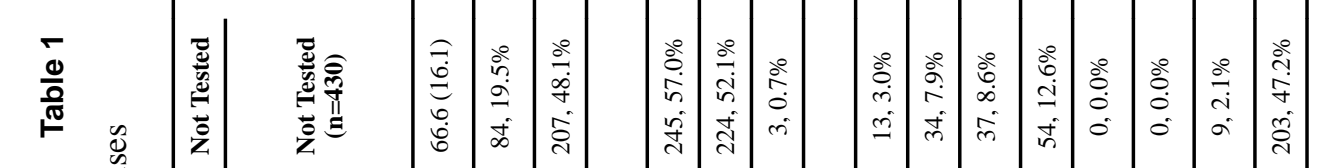

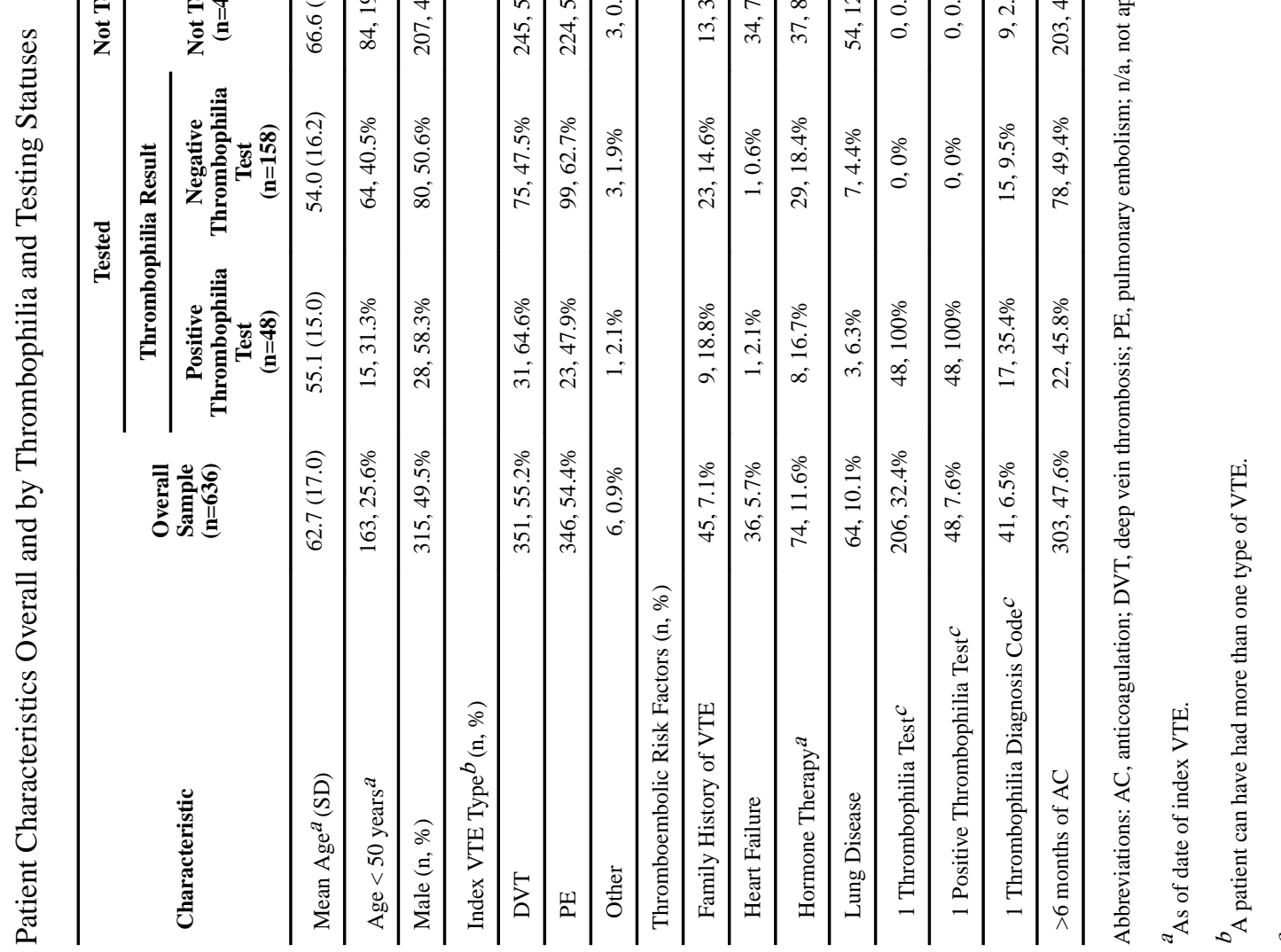

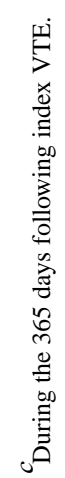


Table 2

Algorithm Performance Results and Statistics ${ }^{a}$

\begin{tabular}{|c|c|c|c|c|}
\hline & $\begin{array}{c}\text { Sensitivity } \\
\% \\
(95 \% \text { CI })\end{array}$ & $\begin{array}{c}\text { PPV } \\
\% \\
(95 \% \mathrm{CI})\end{array}$ & $\begin{array}{c}\text { Specificity } \\
\% \\
(95 \% \text { CI) }\end{array}$ & $\begin{array}{c}\text { NPV } \\
\% \\
(95 \% \text { CI })\end{array}$ \\
\hline ICD-9 & $\begin{array}{c}35.4 \\
(22.2-50.5)\end{array}$ & $\begin{array}{c}41.5 \\
(26.3-57.9)\end{array}$ & $\begin{array}{c}95.9 \\
(94.0-97.4)\end{array}$ & $\begin{array}{c}94.8 \\
(92.7-96.4)\end{array}$ \\
\hline Extended AC & $\begin{array}{c}45.8 \\
(31.4-60.8)\end{array}$ & $\begin{array}{c}7.3 \\
(4.6-10.8)\end{array}$ & $\begin{array}{c}52.2 \\
(48.1-56.3)\end{array}$ & $\begin{array}{c}92.2 \\
(88.8-94.8)\end{array}$ \\
\hline ICD-9 \& Extended AC & $\begin{array}{c}18.8 \\
(9.0-32.6)\end{array}$ & $\begin{array}{c}34.6 \\
(17.2-55.7)\end{array}$ & $\begin{array}{c}97.1 \\
(95.4-98.3)\end{array}$ & $\begin{array}{c}93.6 \\
(91.4-95.4)\end{array}$ \\
\hline
\end{tabular}

Abbreviations: AC, anticoagulation; CI, confidence interval; ICD-9, International Classification of Diseases $9^{\text {th }}$ Revision; NPV, negative predictive value; PPV, positive predictive value.

${ }^{a}$ Patients with a positive thrombophilia laboratory test result were considered truly positive for thrombophilia. Patients with a negative thrombophilia test result and patients who did not undergo thrombophilia testing were considered truly negative for thrombophilia. 